\title{
A Polymerase Chain Reaction-based Linkage Map of Broccoli and Identification of Quantitative Trait Loci Associated with Harvest Date and Head Weight
}

\author{
Allan F. Brown ${ }^{1}$ \\ Department of Natural Resources and Environmental Sciences, University of Illinois, Urbana, \\ IL 61801 \\ Elizabeth H. Jeffery \\ Department of Food Science and Human Nutrition, University of Illinois, Urbana, IL 61801 \\ John A. Juvik ${ }^{2}$ \\ University of Illinois, Natural Resources and Environmental Sciences, 307 Edgar R. Madigan \\ Laboratory, 1201 West Gregory Drive, Urbana, IL 61801
}

\begin{abstract}
AdDitional INDEX WORDs. Brassica oleracea ssp. italica, simple sequence repeat, sequence-related amplified polymorphism, maturity, linkage mapping, QTL

Abstract. A set of 216 polymerase chain reaction-based molecular markers was screened for polymorphisms using two morphologically dissimilar broccoli (Brassica oleracea L. ssp. italica Plenck) lines, 'VI-158' and 'Brocolette Neri E. Cespuglio'. Fifty-nine of these simple sequence repeat (SSR) and sequence-related amplified polymorphic (SRAP) primer pairs generated 69 polymorphisms that were used to construct a linkage map of broccoli from a population of $162 \mathrm{~F}_{2: 3}$ families derived from the cross between these two lines. Ten linkage groups were generated that spanned a distance of $468 \mathrm{cM}$ with an average interval width of $9.4 \mathrm{cM}$. The map was used to identify quantitative trait loci (QTL) associated with differences in harvest date maturity and head weight in the population grown in the same location over 2 years. Heritability estimates for days to maturity and head weight were 0.84 and 0.64 , respectively. Four QTL for harvest maturity were identified that described $55.6 \%$ of the phenotypic variation in the first year with two of these QTL also detected in the second year of the experiment that described $29.2 \%$ of the phenotypic variation. Five QTL were identified as associated with head weight in 1999 and accounted for $71.8 \%$ of the phenotypic variability. Two of these QTL accounted for $24 \%$ of the phenotypic variability in head weight in 2000 . To our knowledge, this is the first linkage map of broccoli and the first combined SSR and SRAP map of B. oleracea, which should provide a useful tool for the genetic analysis of traits specific to ssp. italica.
\end{abstract}

One of the principal problems associated with broccoli production is the lack of harvest time predictability (Hulbert and Orton, 1984). Also, most broccoli breeding programs concentrate on developing early-maturing cultivars, which does not allow for continuity of production throughout the season (Crisp et al., 1986). Maturity among cruciferous vegetables is considered a quantitatively inherited trait that can be influenced by a number of environmental factors such as plant density, temperature, and various horticultural practices (Salter and Fradgley, 1969; Salter and James, 1975; Weibe, 1975). A study of 14 broccoli inbreds grown over seven environments reported that mean maturity date was a trait with relatively high heritability $\left(\mathrm{H}^{2}=0.56\right)$ and was primarily a consequence of additive gene action (Hulbert and Orton, 1984). The heritability of maturity dates in two full-sib populations developed from

Received for publication 11 Apr. 2006. Accepted for publication 8 Apr. 2007. Funding for this research was provided by the Council for Food and Agricultural Research (C-FAR) grants IDACF 99I-65-4-NRES, IDACF 99D-17-4-NRES, and USDA IFAAFS 00-52102-9695.

Use of trade names does not imply endorsement of the products named or criticism of similar ones not named.

We thank Dr. Mark Farnham of the USDA Vegetable Genetic Center (Charleston, SC) for original seed of VI-158.

${ }^{1}$ Current address: USDA-ARS Western Regional Plant Introduction, Washington State University, Pullman, WA 99164.

${ }^{2}$ Corresponding author. E-mail: juvik@uiuc.edu. open-pollinated cultivars was reported to be 0.62 and 0.75 (Legg and Lippert, 1966).

Little is known about genetic factors influencing maturity in broccoli. Camargo and Osborn (1996) created a B. oleracea mapping population by crossing broccoli to cabbage and successfully identified three quantitative trait loci (QTL) accounting for $54.1 \%$ of the phenotypic variability in flowering time, but curd or head formation in B. oleracea involves a complex retardation in the juvenile growth phase and flowering time alone may not fully explain or be correlated with commercial-stage broccoli maturity.

Existing genetic linkage maps of $B$. oleracea have been generated using restriction fragment length polymorphic (RFLP) markers with populations developed by crossing parents from different subspecies such as broccoli $\times$ cabbage (B. oleracea ssp. capitata L.) or kale (B. oleracea ssp. acephala DC) $\times$ cauliflower (B. oleracea ssp. botrytis L.) (Bohuon et al., 1996; Camargo, 1994; Kianian and Quiros, 1992; Lan and Patterson, 2001; Slocum et al., 1990). Crosses between these interfertile $B$. oleracea subspecies maximize genetic variability in the subsequent mapping populations but result in a range of morphological types that form a continuum between the phenotypes of the parents. These populations have been used extensively to characterize traits common to all $B$. oleracea subspecies such as self-incompatibility and host plant disease resistance (Camargo et al., 1995, 1997) but are of lesser value in 
regard to commercially important subspecies-specific traits such as head or curd characteristics of broccoli and cauliflower or the lateral or terminal vegetative budding associated with brussel sprouts (B. oleracea ssp. gemmifera Zenker) or cabbage. Pelofske and Baggett (1979), for example, reported that in a population created by crossing broccoli and cabbage, only $2 \%$ of the $\mathrm{F}_{2}$ plants produced cabbage-like heads and none of these were of commercial grade.

The development of a linkage map specific to broccoli using polymerase chain reaction (PCR)-based markers could facilitate the development of new, superior broccoli inbreds and hybrid cultivars through such strategies as marker-assisted selection and marker-assisted backcrossing. This would reduce the time required to introgress favorable alleles into existing elite germplasm. A number of PCR-based molecular marker systems have been developed in recent years that share a number of common advantages over RFLPs: they are more readily amenable to automation, often produce more polymorphisms per reaction, require less DNA, and can often be multiplexed, resulting in increased savings to researchers. Microsatellite or simple sequence repeat (SSR) markers originally isolated from Arabidopsis thaliana (L.) Heynh. and Brassica napus L. libraries have been used successfully in $B$. oleracea to estimate genetic diversity among and within $B$. oleracea subspecies and a limited number has been added to existing RFLP maps (Plieske and Struss, 2001; Westman and Kresovich, 1998). In Brassica, the distance between these repetitive sequences is estimated to be approximately one in every 100 to $400 \mathrm{~kb}$ DNA (Kresovich et al., 1995; Uzunova and Ecke, 2001). Recently, Lowe et al. (2004) developed and characterized a set of 398 SSR markers from small-insert genomic libraries of Brassica rapa L., Brassica nigra L, $B$. napus, and $B$. oleracea. These markers produce products in both their species of origin and in many cases related Brassica L. species. A genetic linkage map of B. napus was constructed using 97 of these markers that detected 136 loci over 19 linkage groups (Lowe et al., 2004). To date, however, these markers have not been used to construct a linkage map of $B$. oleracea.

Sequence-related amplified polymorphism is a relatively new PCR-based marker system that uses forward and reverse oligonucleotide primers consisting of sequences of no specific constitution ("filler") followed by a core region (comprised of the nucleotides CCGG in the forward primer and AATT in the reverse) and a terminal region of three selective nucleotides (Li and Quiros, 2001). As the authors explain, designing primers with these specific core regions should allow for the targeting of open reading frames because exons tend to be richer than introns in these specific nucleotides. The objectives of this research were to use a subset of these SSR and sequencerelated amplified polymorphic (SRAP) markers to construct a genetic linkage map specific to broccoli and to identify QTL associated with harvest date maturity and broccoli head size.

\section{Materials and Methods}

Plant material. A broccoli population was developed by crossing 'BNC' (a brocolette neri type; accession PI 462209 of the U.S. Department of Agriculture Plant Genetic Resource Unit, Geneva, NY, courtesy of D. Sharman) with 'VI-158,' a calabrese-type double haploid derived from the $F_{1}$ hybrid 'Viking' (courtesy of M. Farnham). Brocolette neri ("black broccoli"') is phenotypically different from calabrese in that it has highly branched dark green leaves, white flowers, and produces multiple small nonuniform-sized heads (Gray, 1993). The resulting population was segregated for a number of morphological and agronomic characteristics, including maturity, head size, branching patterns, waxy leaves, and leaf shape and color.

A single $F_{1}$ plant was self-pollinated to produce $162 F_{2}$ plants and these were subsequently bud-pollinated to produce sufficient $\mathrm{F}_{3}$ seed for DNA extraction and 2 years of replicated field trials. Approximately 24 plants of each $\mathrm{F}_{2: 3}$ family were seeded in the greenhouse 10 June 1999 and 17 June 2000. After 1 week of acclimation in the coldframe, seedlings were transplanted into plots at the University of Illinois South Farm in Champaign on 22 July 1999 and 26 July 2000. As a result of the limited number of seeds, only 84 families were replicated in 2000. Maturity was not a factor in seed set because $F_{2}$ plants were harvested for $\mathrm{F}_{3}$ seed in the greenhouse over multiple years. Soil type was a Drummer silty clay loam. Approximately 4 weeks after transplanting, plants were side-dressed with $13 \mathrm{~N}-$ $5.7 \mathrm{P}-6.6 \mathrm{~K}-8.5 \mathrm{~S}$ granular fertilizer at a rate $17 \mathrm{~kg} \cdot \mathrm{ha}^{-1}$. Standard broccoli cultivation practices were followed with pesticides applied as needed.

Field design in both years was a randomized complete block with two replicates of $\approx 12$ plants spaced $0.3 \mathrm{~m}$ apart with $0.9 \mathrm{~m}$ between rows. At commercial maturity, heads were harvested from each plot and packed in ice for transport to the laboratory. As $\mathrm{F}_{2: 3}$ families were segregating for maturity, multiple harvests were done whenever three or more individuals from each family were ready for harvest. Each plot was harvested at least twice. Harvest date was computed using the formula $\left[\mu\left(\mathrm{h}_{i j} / \mathrm{n}_{i j}\right)\right] / \mathrm{N}_{i}$, in which $\mathrm{h}_{i j}$ is the number of days from transplant to harvest for family $i$ on harvest date $j, \mathrm{n}_{i j}$ is the number of heads from family $i$ on harvest date $j$, and $\mathrm{N}_{i}$ is the total number of heads harvested from family $i$ overall harvest dates. Heads were cut $\approx 3 \mathrm{~cm}$ below where the branches of central inflorescence joined together and head weight was recorded. Head weight for each family/replicate was based on data averaged from six to 12 plants. Plants were determined to be selfcompatible if they set seed freely without the need for bud pollination. Because approximately one-fourth of all plants produced thousands of seeds, the trait was treated as if it were controlled by a single gene.

DNA EXTRACTION. Leaves of at least 12 plants from each $\mathrm{F}_{2: 3}$ family were harvested at $\approx 6$ weeks of growth and were kept at $-80{ }^{\circ} \mathrm{C}$ until lyophilized. Freeze-dried tissue was ground to a fine powder with liquid nitrogen and DNA was extracted using a modified CTAB procedure as described by Kidwell and Osborn (1992). To minimize contamination from polysaccharides that might interfere with subsequent manipulations, DNA was resuspended in high-salt TE buffer (10 mM Tris, $\mathrm{pH} 8.0$, $1 \mathrm{~mm}$ EDTA, pH 8.0, $1 \mathrm{M} \mathrm{NaCl})$ and stored at $4{ }^{\circ} \mathrm{C}$ overnight. DNA was reprecipitated the next morning with two-thirds volume of isopropanol.

Polymerase chain reaction fragment analysis. One hundred sixty-one SSR primer sequence pairs were obtained from the public database BrassicaDB, a database component of UK Cropnet (Dicks et al., 2000; Lowe et al., 2004). SSR primers were derived from BAC libraries constructed from the following species: B. oleracea (92 primer pairs), B. napus (49 primer pairs), B. rapa (13 primer pairs), and $B$. nigra (seven primer pairs). Designation of these primers reflects their library 
of origin: $\mathrm{OL}=$ oleracea, $\mathrm{NA}=$ napus, $\mathrm{NI}=$ nigra, and $\mathrm{RA}=$ rapa. Forward and reverse oligonucleotides corresponding to these sequences were synthesized by Operon Technologies (Alameda, CA).

Thirty SSR primer sequence pairs designated BMS, previously mapped in B. oleracea (Saal et al., 2001), were obtained from Plant Gene Track (Goettingen, Germany) and five forward and six reverse primers corresponding to published SRAP sequences (Li and Quiros, 2001) were synthesized by Applied Biosystems (Foster City, CA). SRAP designation includes the specific forward and reverse primers used to generate the polymorphism. In the case of multiple polymorphisms from a single combination of SRAP primers, ascending alphabetic letters are used to designate the fragment's relative size (i.e., M4E4a is a smaller-sized fragment than M4E4b).

PCR amplification was conducted using MJ PTC-100 or MJ PTC-400 thermocyclers (MJ Research, Watertown, MA). PCR reaction mixes contained $30 \mathrm{ng}$ of genomic template DNA in $3 \mu \mathrm{L}$ of $1 \times \mathrm{TE}$ and $12 \mu \mathrm{L}$ of PCR master mix (1× PCR buffer, $2.5 \mathrm{~mm} \mathrm{MgCl}, 0.4 \mathrm{~mm}$ each of dATP, dCTP, dGTP, dTTP, $20 \mu \mathrm{M}$ forward and reverse primer, and 0.75 Units of Taq polymerase). Cycling conditions for SSR and SRAP amplification included an initial 4-min denaturation step at $94{ }^{\circ} \mathrm{C}$ followed by 35 cycles that consisted of $45 \mathrm{~s}$ denaturation at $94{ }^{\circ} \mathrm{C}, 45 \mathrm{~s}$ annealing at $50{ }^{\circ} \mathrm{C}$, and $1 \mathrm{~min}$ elongation at $72^{\circ} \mathrm{C}$. The final cycle included a 7 -min elongation step. A subset of lines, including parents, $\mathrm{F}_{1}$, and five randomly chosen $\mathrm{F}_{2: 3}$ lines, were included in the initial screen to identify useful polymorphisms.

Primer pairs that generated polymorphic bands in the initial screen of 'BNC' and 'VI-158' were selected for analysis of the $F_{2: 3}$ population. Primer pairs were resynthesized with the forward primer labeled with a blue (6-FAM), green (HEX), or yellow (NED) fluorescent tag (Applied Biosystems). Detection of PCR fragments was accomplished with a $4.8 \%$ polyacrylamide gel [14.4 g urea, $4.8 \mathrm{~mL}$ (19:1) acrylamide/ bisacrylamide, $1 \mathrm{~g}$ of Amberlite resin (Sigma-Aldrich, St. Louis), $22 \mathrm{~mL}$ of distilled $\mathrm{H}_{2} \mathrm{O}$ ] using an $\mathrm{ABI} 377$ automated sequencer (Applied Biosystems) following the manufacturer's protocol. Length of the run was 2.5 and $3.0 \mathrm{~h}$ for SSR and SRAP markers, respectively. Depending on the efficiency of amplification, 1 to $2 \mu \mathrm{L}$ of the PCR reaction was added to a loading mix that consisted of $250 \mu \mathrm{L}$ of deionizied formamide, $25 \mu \mathrm{L}$ GeneScan 500 size standard, and $25 \mu \mathrm{L}$ Gene-Scan loading buffer.

Scoring of the fragments was accomplished using GeneScan software from Applied Biosystems. In addition to the PCR markers, two phenotypic characteristics, flower color (FLWR) and self-compatibility (SC), were scored as morphological markers from field and greenhouse data. Analysis was conducted at the Illinois Genetic Marker Center, University of Illinois, Urbana.

STatistical analysis. Linkage map was constructed using the Kosambi mapping function of the JOINMAP program (van Ooijen and Voorrips, 2001). An independence logarithm of odds (LOD) score of 3.0 was required for grouping of the markers and the order of the markers on each linkage group was determined using a minimum LOD of 1.0 and a maximum recombination frequency of 0.4 . LOD scores generated by Joinmap use a G2 statistic for independence that, unlike LOD scores normally used in linkage analysis, results in reduced incidents of spurious linkage in the presence of segregation distortion (van Ooijen and Voorrips, 2001). Putative QTL were detected with MapQTL (van Ooijen et al., 2002), which uses a maximum likelihood approach to generate a multiple QTL model with additive and dominance effects. Cofactors were selected for each trait by first performing a simple interval analysis and identifying flanking markers that exceeded a LOD threshold of 2.5. In the multiple QTL model, an LOD score of 3.0 was used to declare a putative QTL. This level was chosen to minimize type II errors and because we were principally interested in those QTL detected in both years because these are presumably most likely to be stable. As a result of the limited number of genotypes in the second year, each year was analyzed separately. As is common practice, estimates of QTL location were obtained where the LOD score assumes its maximum and support intervals represent 1.0 LOD dropoff on either side of this position. The ratio of dominance to additivity (d/a) was used to determine the type of gene action. Gene action is described as additive if $\mathrm{d} / \mathrm{a}$ is 0 to 0.2 , partially dominant if $\mathrm{d} / \mathrm{a}$ is from 0.21 to 0.80 , dominant if $\mathrm{d} / \mathrm{a}$ is from 0.81 to 1.20 , and overdominant if $\mathrm{d} / \mathrm{a}$ is greater than 1.20 (Stuber et al., 1987).

\section{Results}

Maturity AND head Weight Data. Average maturity among the $\mathrm{F}_{2: 3}$ families in 1999 was $72 \mathrm{~d}$ with a range from 45 to $109 \mathrm{~d}$ (Table 1). Average maturity among $\mathrm{F}_{2: 3}$ families was earlier in 2000 with a mean of $66 \mathrm{~d}$ and a range of 49 to $101 \mathrm{~d}$. Standard deviation for 1999 and 2000 was 12.5 and 12.3, respectively. 'VI-158' was consistently among the last genotypes to be harvested. 'BNC' was less predictable, but the average harvest was $21 \mathrm{~d}$ earlier than 'VI-158' in 1999 and $16 \mathrm{~d}$ earlier in 2000. Significant transgressive segregation occurred toward early maturity with more than half of families in both years harvested before the earliest maturing parent.

Average head weight in 1999 was $71.5 \mathrm{~g}$ with a range from $12.9 \mathrm{~g}$ to $245 \mathrm{~g}$. Average head weight was smaller in 2000 with an average weight of $49.2 \mathrm{~g}$ and a range of 12.4 to $117 \mathrm{~g}$. The difference between mean head weight between the two seasons can be attributed in part to the weather. The 1999 growing season provided superior conditions for growth and flowering in broccoli with greater than average rainfall and substantially cooler temperatures in July and August. In contrast, 2000 was noted for below normal precipitation and above normal temperatures in July and August. No significant transgressive segregation was observed for head weight, because 'BNC' and

Table 1. Mean, SD $\left(\sigma^{2}\right)$, and range for head weight (grams fresh weight), maturity (days from transplant to harvest) of parental lines, $\mathrm{F}_{1}$, and derived $\mathrm{F}_{2: 3}$ broccoli families from the cross VI-158 $\times$ Brocolette Neri E. Cespuglio (BNC).

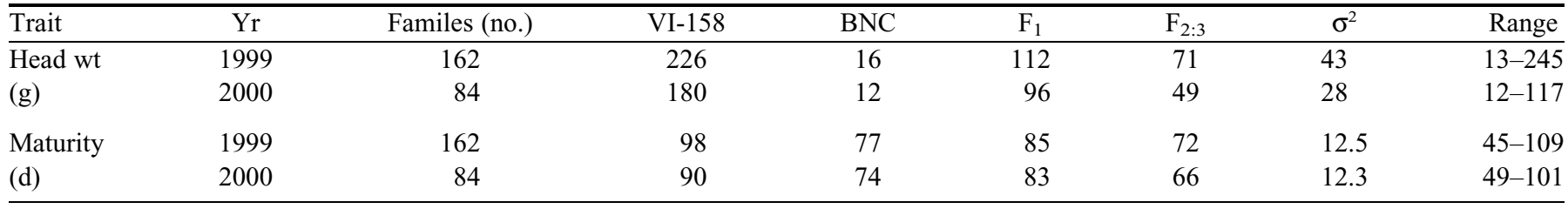


'VI-158' were among the smallest and largest heads measured in both years. Maturity ratings and head weight did not fit a normal distribution, and attempts to improve normality using standard transformations were not successful. It should be noted, however, that harvesting of broccoli continued until mid-November in both years until temperatures fell below $-2{ }^{\circ} \mathrm{C}$, and it is expected that some of the late-maturing families were not as well represented as earlier maturing families. At least one harvest per family was recorded in both years and neither year's harvest was truncated as a result of less than optimal conditions.

Primer SCReEning. Results from the initial screening of the primers are presented in Table 2. Primer pair sequences obtained from BrassicaDB (Dicks et al., 2000) identified 21 primer pairs $(13 \%)$ that failed to produce a PCR fragment, 99 primer pairs $(62 \%)$ that produced one or more monomorphic fragments, and 41 primer pairs $(25 \%)$ that produced at least one polymorphic fragment. As expected, higher rates of polymorphism were detected using primer sequences isolated from the $B$. oleracea genomic library $(35 \%)$ than using sequences derived from $B$. napus and $B$. rapa (16\% and $8 \%$, respectively). Of the seven primer pairs originating from $B$. nigra, three produced a monomorphic single band and four failed to amplify sequences from either parent.

All 30 of the BMS SSR primer pairs produced at least one PCR fragment and $13(43 \%)$ identified one or more polymorphisms. Thirteen of the 25 combinations of the forward and reverse SRAP primers produced at least one polymorphic dominant band. Up to four polymorphisms were detected with

Table 2. Results of the screening of simple sequence repeat and sequence related amplified polymorphic primer pairs for polymorphism in an $\mathrm{F}_{2: 3}$ population of broccoli derived from the cross VI-158 $\times$ Brocolette Neri E. Cespuglio.

\begin{tabular}{lc}
\hline Source of markers & $\begin{array}{c}\text { Markers } \\
\text { [no. }(\% \text { of total)] }\end{array}$ \\
\hline SSR & 161 \\
BrassicaDb $^{z}$ & 21 \\
No amplification & 99 \\
Monomorphic & $41(25 \%)$ \\
Polymorphic & $32(35 \%)$ \\
Brassica oleracea $(92)^{y}$ & $1(8 \%)$ \\
Brassica rapa $(13)$ & $8(16 \%)$ \\
Brassica napus $(49)$ & 0 \\
Brassica nigra $(7)$ & 30 \\
BMS & 17 \\
Monomorphic & $13(43 \%)$ \\
Polymorphic & 25 \\
SRAPw & 12 \\
Monomorphic & $13(52 \%)$ \\
Polymorphic & 216 \\
Total & $21(10 \%)$ \\
No amplification & $128(59 \%)$ \\
Monomorphic & $67(31 \%)$ \\
Polymorphic &
\end{tabular}

$\overline{{ }^{\mathrm{z}} \text { Simple sequence repeat (SSR) primer sequences obtained BrassicaDb }}$ (Dicks et al., 2000).

${ }^{\mathrm{y}}$ Number of BrassicaDb primers screened from genomic libraries by species.

xPrimers obtained Plant Gene Track (Goettingen, Germany) (Saal et al., 2001).

${ }^{\text {w}}$ Sequence-related amplified polymorphism ( $\mathrm{Li}$ and Quiros, 2001). a single primer combination and on average, 1.7 polymorphisms were detected per combination. In total, 67 PCR-based markers identified one or more polymorphisms.

LINKAGE MAP. Of the 67 polymorphic markers identified in the initial screen, 55 primer pairs generated a total of 69 reliable polymorphisms in the mapping population. With the inclusion of the two phenotypic markers, FLWR and SC, the total number of markers used to generate the map was 71. All of the SRAP and 21 of the SSR loci were scored as dominant markers. FLWR and SC were scored as codominant and dominant markers, respectively.

Ten linkage groups were generated spanning $468 \mathrm{cM}$ with an average interval length of $9.4 \mathrm{cM}$. These linkage groups are designated A, B, C, D, E, F, G, H, I, and J. Sixty-two loci were successfully mapped with nine loci (six SSR, two SRAP, and SC) unlinked (Fig. 1). Linkage groups ranged in size from 9 to $97 \mathrm{cM} . \chi^{2}$ analysis revealed 17 loci with segregation ratios that deviated significantly $(P<0.05)$ from the expected Mendelian ratios $(25 \%)$. Segregation distortion was primarily confined to three regions on linkage groups B, C, and E and within these regions, flanking markers tended to reflect an overrepresentation of alleles from the same parent. Markers within these regions included both SSR and SRAP markers.

IDENTIFICATION OF QUANTITATIVE TRAIT LOCI ASSOCIATED WITH MATURITY AND HEAD WEIGHT. Four QTL on linkage groups A, D, E, and I (designated MAT-1 through MAT-4) accounted for $55.6 \%$ of the phenotypic variability in broccoli maturity in 1999 (Table 3; Fig. 1). Alleles associated with earlier maturity were contributed by both 'BNC' (MAT-1 and MAT-2) and 'VI$158^{\prime}$ (MAT-3 and MAT-4). Gene action was predominantly additive with the exception of MAT-1 that displayed overdominance. MAT-2 and MAT-3 had the largest individual effects, each contributing $\approx 20 \%$ to the phenotypic variability.

In 2000, only $M A T-2$ was significant at $\mathrm{LOD}=3.0$; however, MAT-1 had the second highest LOD score in that year (LOD = 2.58) and when data from the same families grown in both years were averaged, both MAT-1 and MAT-2 were significant (Table 3). Together, MAT-1 and MAT-2 described $29.2 \%$ of the phenotypic variability in 2000 and $34.5 \%$ of the variation when maturity scores are averaged over the 2 years.

Five QTL on linkage groups D, E, F, H, and I (designated $H W-1$ through $H W-5$ ) were significantly associated with head weight in 1999 and explained $71.8 \%$ of the phenotypic variance (Table 4). All alleles associated with increased head weight were contributed by 'VI-158' and all alleles were predominately additive except $H W-2$ (dominant). Effects of the individual alleles ranged from $8.1 \%$ to $23.7 \%$. In 2000 , only $H W-4$ was significant at $\mathrm{LOD}=3.0$; however, $H W$-3 had the second highest LOD in that year $(\mathrm{LOD}=2.63)$ and together the two QTL explained $24 \%$ of the phenotypic variance.

\section{Discussion}

An estimate of the expected size of the broccoli genome can be generated by averaging the size of the $B$. oleracea linkage maps reported by Bohuon et al. (1996), Kianian and Quiros (1992), and Slocum et al. (1990). On the basis of this estimate, our map covers $\approx 70 \%$ of the genome. It is expected that regions affecting traits of interest may fall outside the span of our markers and not be detected.

In the current study, 67 of 216 markers (31\%) amplified polymorphic products. This is in contrast to polymorphic rates 

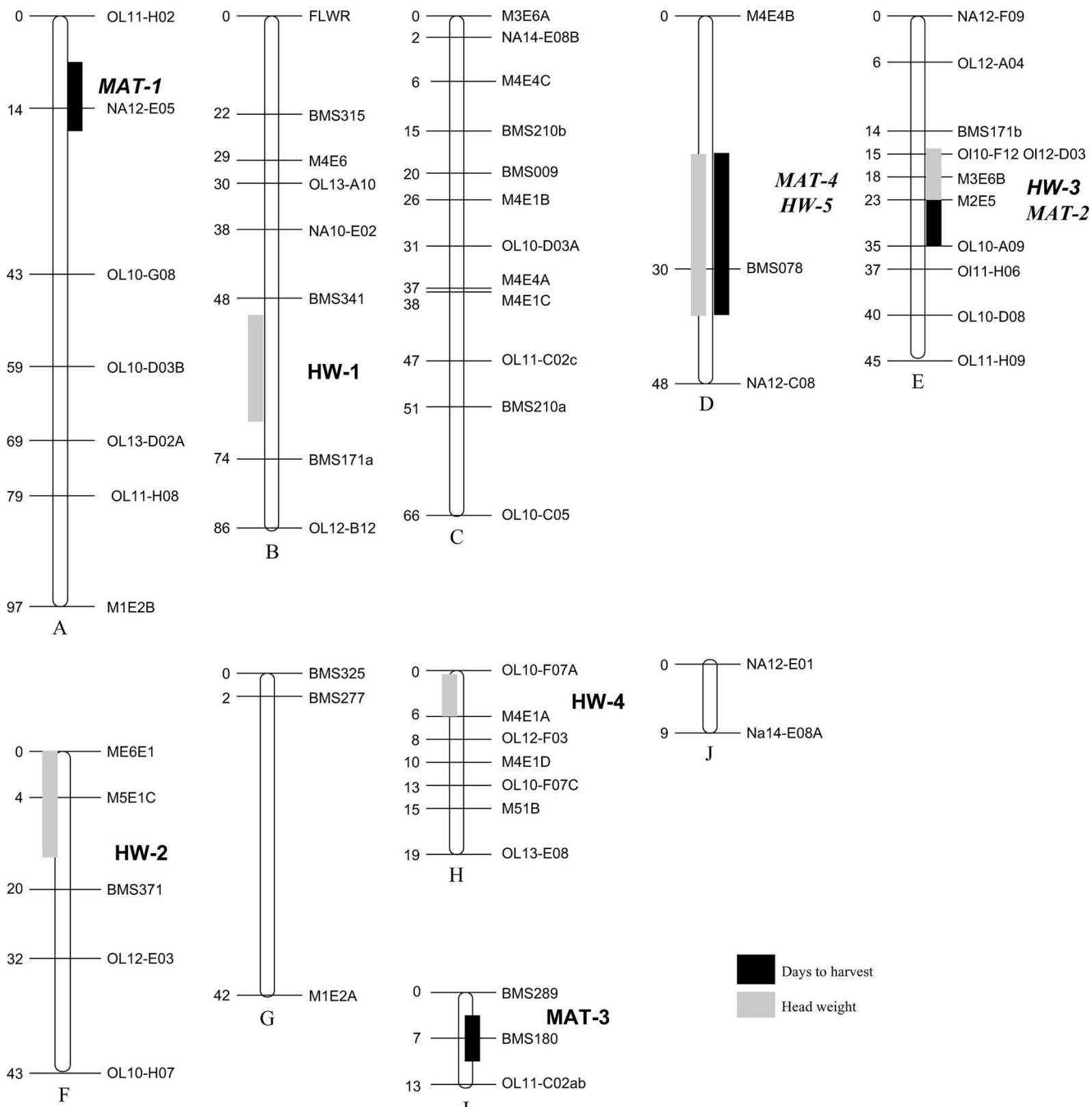

HW-4
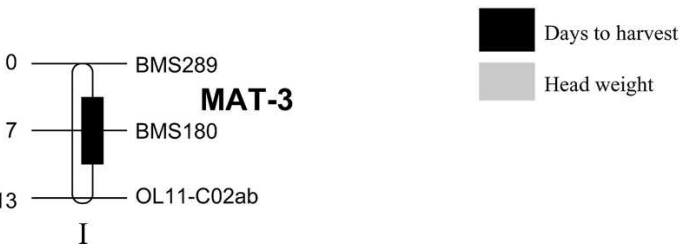

Fig. 1. Genetic linkage map of broccoli and quantitative trait loci associated with maturity and head weight.

of between $45 \%$ and $85 \%$ for $B$. oleracea mapping populations generated using parents of different subspecies and suggests a lack of genetic variability in this particular cross and perhaps within ssp. italica. Despite this limitation, however, the use of the map and the mapping population is supported by the results of the QTL analysis that described $71.8 \%$ and $55.6 \%$ of the phenotypic variance associated with head weight and maturity in the first year of analysis. The limited number of genotypes in the second year reduced the power of the experiment to identify QTL with lesser effects and in light of this, the second year should not be viewed as a replication but rather as a validation set to provide further support for the QTL identified in the first year. The second year's data were useful in providing support for the importance of the $M A T-1$ and $M A T-2$ loci that together described almost $30 \%$ of the phenotypic variability in maturity and for $H W-3$ and $H W-4$ that described $25 \%$ of the variability for head weight in both years.
A comparison between the map described in this article and that of Lowe et al. (2004) (Fig. 2) detected a homologous region between $B$. oleracea and B. napus. Markers OL11-H02, NA12-E05, OL10-G08, OL13-D02A, and OL11-H08 span a region of $79 \mathrm{cM}$ on linkage group $\mathrm{A}$ in our map. These five markers also occur in the same sequential order on B. napus chromosome N14 and span a distance of $\approx 73.9 \mathrm{cM}$ (Lowe et al., 2004) (Fig. 2). Because B. oleracea is a progenitor species of the amphidiploid B. napus (Prakash and Hinata, 1980; U, 1935), the colinearity of these linkage groups could represent a conserved region between the species and could provide an opportunity for further genetic analysis of $M A T-1$, which occurs in this region. Parkin et al. (2002) identified regions of colinearity between $A$. thaliana chromosome 5 and B. napus chromosomes N2, N3, N10, N12, N13, and N19. This region on $A$. thaliana chromosome 5 contains $F L C$, a gene that has been implicated in regulation of flowering time in Brassica 
Table 3. Putative quantitative trait loci (QTL) associated with maturity in $\mathrm{F}_{2: 3}$ families from the broccoli cross VI-158 $\times$ Brocolette Neri E. Cespuglio in 1999 and 2000.

\begin{tabular}{|c|c|c|c|c|c|c|c|c|c|}
\hline \multirow{2}{*}{$\frac{\overline{\text { QTL }}}{M A T-1}$} & & $\mathrm{LG}^{\mathrm{z}}$ & Pos. $(\mathrm{cM})^{\mathrm{y}}$ & $\mathrm{LOD}^{\mathrm{x}}$ & $\mathrm{CFM}^{\mathrm{w}}$ & $\% \sigma_{\mathrm{p}}^{\mathrm{v}}$ & Parental source $^{\mathrm{u}}$ & Add. $(\mathrm{g})^{\mathrm{t}}$ & $\overline{\text { Dom. }(\mathrm{g})^{\mathrm{s}}}$ \\
\hline & 1999 & A & 10.0 & 6.01 & NA12-E05 & 9.0 & $\mathrm{BNC}$ & 4.47 & 6.17 \\
\hline$M A T-2$ & 1999 & $\mathrm{E}$ & 33.0 & 6.57 & OL10-A09 & 18.8 & VI-158 & 7.76 & 1.06 \\
\hline \multirow[t]{2}{*}{$M A T-3$} & 1999 & $\mathrm{~J}$ & 18.0 & 4.72 & BMS078 & 7.9 & VI-158 & 4.94 & 2.62 \\
\hline & 2000 & $\mathrm{~J}$ & - & - & - & - & - & - & - \\
\hline$M A T-4$ & 1999 & $\mathrm{D}$ & 13.0 & 7.33 & $\mathrm{OL} 11-\mathrm{C} 02$ & 19.9 & VI-158 & 6.48 & 2.52 \\
\hline
\end{tabular}

${ }^{\mathrm{z}}$ Linkage group (see Fig. 1).

${ }^{y}$ Most likely position from end of linkage group determined by maximum LOD score.

${ }^{\mathrm{x}}$ Maximum logarithm of odds score.

${ }^{\mathrm{w}}$ Closest flanking marker.

${ }^{v}$ Percent phenotypic variance explained by QTL.

uparental source of allele that increased days to harvest.

${ }^{t}$ Additive effect (in days), 1/2(QQ-qq).

${ }^{\mathrm{s}}$ Dominant gene action (in days), $1 / 2[(2 \mathrm{Qq})-\mathrm{QQ}-\mathrm{qq}]$.

Table 4. Putative quantitative trait loci associated with head weight (grams fresh weight) in $\mathrm{F}_{2: 3}$ families from the broccoli cross $\mathrm{VI}-158 \times$ Brocolette Neri E. Cespuglio in 1999.

\begin{tabular}{|c|c|c|c|c|c|c|c|c|c|}
\hline$\overline{\mathrm{QTL}}$ & $\mathrm{Yr}$ & $\mathrm{LG}^{\mathrm{z}}$ & Pos. $(\mathrm{cM})^{\mathrm{y}}$ & $\mathrm{LOD}^{\mathrm{x}}$ & $\mathrm{CFM}^{\mathrm{w}}$ & $\% \sigma_{\mathrm{p}}^{\mathrm{v}}$ & Parental source $^{\mathrm{u}}$ & Add. $(g)^{t}$ & $\overline{\text { Dom. }(\mathrm{g})^{\mathrm{s}}}$ \\
\hline \multirow[t]{2}{*}{$\overline{H W-1}$} & 1999 & B & 22.0 & 5.38 & BMS171 & 12.9 & VI-158 & 19.6 & 10.7 \\
\hline & 一 & - & - & 一 & - & - & - & 一 & 一 \\
\hline$H W-2$ & 1999 & $\mathrm{~F}$ & 0.0 & 5.93 & ME6E1 & 23.7 & VI-158 & 21.0 & 24.4 \\
\hline \multirow[t]{2}{*}{$H W-3$} & 1999 & $\mathrm{E}$ & 18.0 & 5.08 & M3E6B & 8.1 & VI-158 & 20.6 & 5.7 \\
\hline & 2000 & $\mathrm{E}$ & 18.0 & 2.63 & M3E6B & 8.8 & VI-158 & 18.0 & 4.9 \\
\hline$H W-4$ & 1999 & $\mathrm{~F}$ & 0.0 & 6.30 & M5E1A & 14.2 & VI-158 & 20.9 & 2.0 \\
\hline \multirow[t]{2}{*}{$H W-5$} & 1999 & $\mathrm{D}$ & 18.0 & 6.06 & BMS078 & 12.9 & VI-158 & 19.4 & 9.3 \\
\hline & - & - & - & - & - & - & - & - & - \\
\hline
\end{tabular}

${ }^{\mathrm{z}}$ Linkage group (see Fig. 2).

${ }^{\mathrm{y}}$ Most likely position from end of linkage group determined by maximum LOD score.

${ }^{\mathrm{x}}$ Maximum logarithm of odd score.

${ }^{\mathrm{w}}$ Closest flanking marker.

vercent phenotypic variance explained by quantitative trait loci (QTL).

"Parental source of allele that increased head weight.

${ }^{\mathrm{t}}$ Additive effect (in grams), 1/2(QQ-qq).

${ }^{\mathrm{s}}$ Dominant gene action (in grams), 1/2[(2Qq)-QQ-qq].

species (Schranz et al., 2002). No genes affecting flowering time have been described on B. napus chromosome N14, which would suggest that the MAT-1 may affect head maturity in a manner not directly related to floral induction. As a result of lack of common markers between the mapping populations, it is difficult at present to ascertain if MAT-2, MAT-3, or MAT-4 corresponds to $F L C$ homologs previously described in B. oleracea populations (Camargo and Osborn, 1996; Schranz et al., 2002).

Six of the linkage groups generated in this study can be aligned to an existing $B$. oleracea linkage map on the basis of common markers. The 'BMS' SSR markers were previously mapped in a cauliflower $\times$ collard $(B$. oleracea $\mathrm{ssp}$. sabellica L.) population (Saal et al., 2001). BMS210a, BMS009, and BMS210b (linkage group C), BMS325 and BMS277 (linkage group G), BMS289 and BMS180 (linkage group I), and BMS315, BMS341, and BMS171a (linkage group B) all occur on common linkage groups in both our map and the collard $\times$ cauliflower maps, about the same map distance relative to each other. BMS371 and BMS078 (linkage groups $\mathrm{F}$ and $\mathrm{D}$, respectively) occur on distinct linkage groups in both populations. BMS029 was unlinked in the current map, although it mapped to the same linkage group as BMS078 in the previous map. In the collard $\times$ cauliflower population, the BMS029 locus occurs at the distal end of the linkage group, $53 \mathrm{cM}$ from BMS078, and it is not unreasonable to assume that it remained unlinked on our map because it exceeded the allowable recombination distance from the nearest marker.

This is the first report of a broccoli linkage map and of QTL associated with traits specific to broccoli. The $\mathrm{F}_{2: 3}$ population derived from the cross 'BNC' $\times$ 'VI-158' displayed variability for a number of additional, useful traits, including uniformity of harvest and head quality. Expectations are that this will be a valuable resource to breeders. The development of the linkage map underlines the need to assess broccoli germplasm to determine empirically the extent of diversity within the subspecies of $B$. oleracea. 


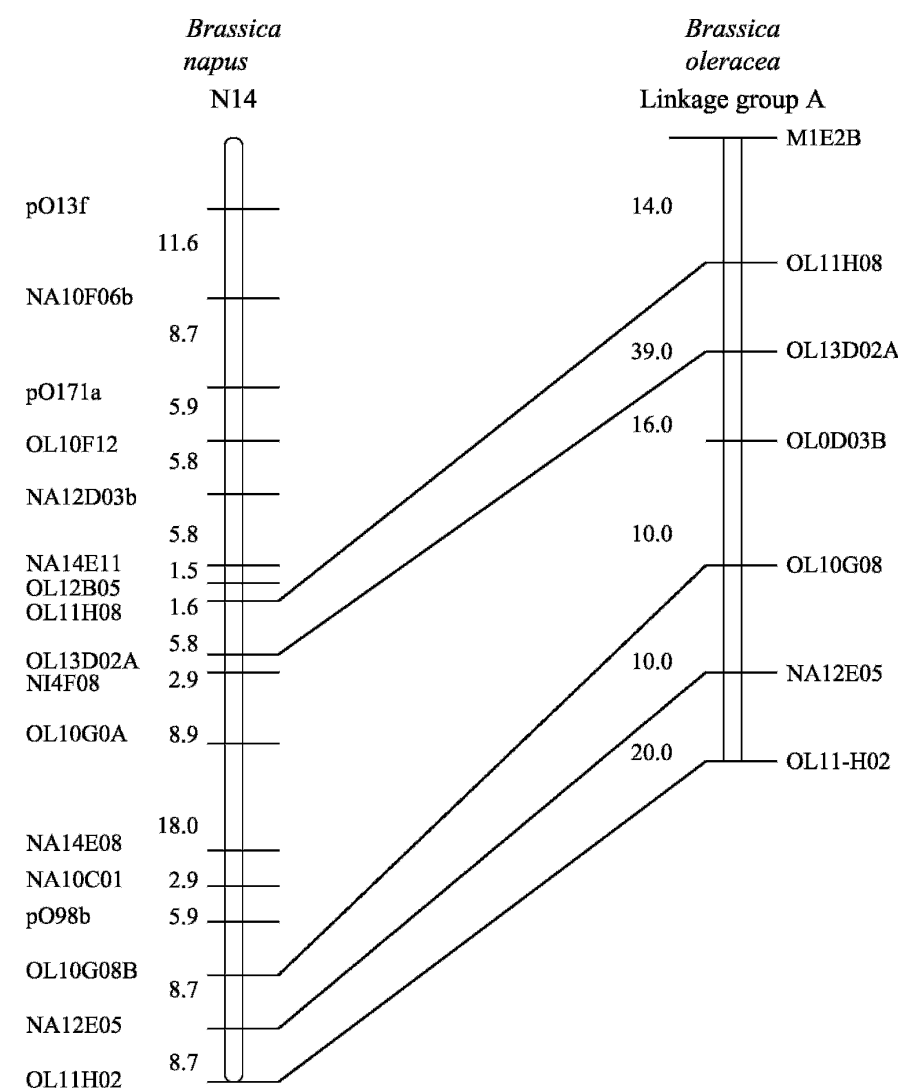

Fig. 2. Comparison of broccoli linkage group D and rapeseed chromosome N14 (Lowe et al., 2004).

\section{Literature Cited}

Bohuon, E.J., D.J. Keith, I.A. Parkin, A.G. Sharpe, and D.Y. Lydiate. 1996. Alignment of the conserved C genomes of Brassica oleracea and Brassica napus. Theor. Appl. Genet. 93:833-839.

Camargo, L.E. 1994. Mapping RFLP and quantitative trait loci in Brassica oleracea. University of Wisconsin, Madison. PhD Thesis.

Camargo, L.E. and T.C. Osborn. 1996. Mapping loci controlling flowering time Brassica oleracea. Theor. Appl. Genet. 92:610-616. Camargo, L.E., L. Savides, G. Jung, J. Nienhuis, and T.C. Osborn. 1997. Location of the self-incompatibility locus in an RFLP and RAPD map of Brassica oleracea. J. Hered. 88:55-60.

Camargo, L.E., P.H. Williams, and T.C. Osborn. 1995. Mapping of quantitative trait loci controlling resistance of Brassica oleracea to Xanthomonas campestris pv. campestris in the field and greenhouse. Phytopathology 85:1296-1300.

Crisp, P., A.R. Gray, S.M. Angell, P.J. Salter, J. Akehurst, and R.A. Sutherland. 1986. The effects of plant spacing on the breeding of broccoli from an expanded genetic base. J. Hort. Sci. 61:205-215.

Dicks, J., M. Anderson, L. Cardle, S. Cartinhour, M. Couchman, G. Davenport, J. Dickson, M. Gale, D. Marshall, S. May, H. Mcwilliam, A. O'Malia, H. Ougham, M. Trick, S. Walsh, and R. Waugh. 2000. UK CropNet: A collection of databases and bioinformatics resources for crop plant genomics. Nucleic Acids Res. 28:104-107.

Gray, A.R. 1993. Broccoli, p. 61-87. In: G. Kallo and B. Bergh (eds.). Genetic improvement of vegetable crops. Pergamon Press, New York. Hulbert, S.H. and T.J. Orton. 1984. Genetic and environmental effects on mean maturity date and uniformity in broccoli. J. Amer. Soc. Hort. Sci. 109:487-490.

Kianian, S.F. and C.F. Quiros. 1992. Generation of a Brassica oleracea composite RFLP map: Linkage arrangements among various populations and evolutionary implications. Theor. Appl. Genet. 84: 544-554.
Kidwell, K.K. and T.C. Osborn. 1992. Simple plant DNA isolation procedures, p. 1-13. In: J.S. Beckman and T.C. Osborn (eds.). Plant genomes: Methods for genetic and physical mapping. Kluwer Academic, Amsterdam, The Netherlands.

Kresovich, S., A.K. Szewc-McFadden, and J.R. McFerson. 1995. Abundance and characterization of simple-sequence repeats (SSRs) isolated from a size-fractionated genomic library of Brassica napus L. (rapeseed). Theor. Appl. Genet. 91:206-211.

Lan, T.H. and A.H. Paterson. 2001. Comparative mapping of QTLs determining the plant size of Brassica oleracea. Theor. Appl. Genet. 103:383-397.

Legg, P.D. and L.F. Lippert. 1966. Genetic variation in openpollinated varieties of broccoli (Brassica oleracea var. italica). J. Hort. Sci. 88:411-416.

Li, G. and C.F. Quiros. 2001. Sequence-related amplified polymorphism (SRAP), a new marker system based on a simple PCR reaction: Its application to mapping and gene tagging in Brassica. Theor. Appl. Genet. 103:455-461.

Lowe, A.J., C.L. Moule, M. Trick, and K.J. Edwards. 2004. Efficient largescale development of microsatellite for marker and mapping applications in Brassica crop species. Theor. Appl. Genet. 108:1103-1112.

Parkin, I.A., D.J. Lydiate, and M. Trick. 2002. Assessing the level of collinearity between Arabidopsis thaliana and Brassica napus for A. thaliana chromosome 5. Genome 45:356-366.

Pelofske, P.J. and J.R. Bagget. 1979. Inheritance of internode length, plant form and annual habit in a cross of cabbage and broccoli (Brassica oleracea var. capitata and var. italica). Euphytica 28:189-197.

Plieske, J. and D. Struss. 2001. Microsatellite markers for genome analysis in Brassica. 1. Development in Brassica napus and abundance in Brassicaceae species. Theor. Appl. Genet. 102:689-694.

Prakash, S. and K. Hinata. 1980. Taxonomy, cytogenetics, and origin of crop Brassica, a review. Opera Botanica 55:1-59.

Saal, B., J. Plieske, J. Hu, C.F. Quiros, and D. Struss. 2001. Microsatellite markers for genome analysis in Brassica: Assignment of rapeseed microsatellites to the $\mathrm{A}$ and $\mathrm{C}$ genomes and genetic mapping in Brassica oleracea L. Theor. Appl. Genet. 102:695-699.

Salter, P.J. and J.R. Fradgley. 1969. Studies on crop maturity in cauliflower: Effects of cultural factors on the maturity characteristics of a cauliflower crop. J. Hort. Sci. 44:141-154.

Salter, P.J. and J.M. James. 1975. The effect of plant density on the initiation, growth and maturity of curds of cauliflower varieties. J. Hort. Sci. 50:239-248.

Schranz, M.E., P. Quijada, S. Sung, L. Lukins, R. Amasino, and T.C. Osborn. 2002. Characterization and effects of the replicated flowering time gene FLC in Brassica rapa. Genetics 162:1457-1468.

Slocum, M.K., S.S. Figdore, W.C. Kennard, J.Y. Suzuki, and T.C. Osborn. 1990. Linkage arrangement of restriction fragment length polymorphism loci in Brassica oleracea. Theor. Appl. Genet. 80:57-64.

Stuber, C.W., M.D. Edwards, and J.F. Wendel. 1987. Molecular-marker facilitated investigations of quantitative trait loci in maize. II. Factors influencing yield and its component traits. Crop Sci. 27:639-648.

$\mathrm{U}, \mathrm{N} .1935$. Genome analysis in Brassica with special reference to the experimental formation of $\mathrm{B}$. napus and its peculiar mode of fertilization. Jpn J. Bot. 7:389-452.

Uzunova, M.I. and W. Ecke. 2001. Abundance, polymorphism and genetic mapping of microsatellites in oilseed rape (Brassica napus L.). Plant Breed. 118:323-326.

van Ooijen, J.W., M.P. Boer, R.C. Jansen, and C. Maliepaard. 2002. MapQTL ${ }^{\circledR} 4.0$, Software for the calculation of QTL position on genetic maps. Plant Research Intl., Wageningen, The Netherlands.

van Ooijen, J.W. and R.E. Voorrips. 2001. JoinMap ${ }^{\circledR}$ version 3.0, Software for the calculation of genetic linkage maps. Plant Research Intl., Wageningen, The Netherlands.

Weibe, H.J. 1975. The morphological development of cauliflower and broccoli cultivars depend on temperature. Sci. Hort. 3:95-101.

Westman, A.L. and S. Kresovich. 1998. The potential for cross-taxa simple-sequence repeat (SSR) amplification between Arabidopsis thaliana L. and crop Brassicas. Theor. Appl. Genet. 96:272-281. 